\title{
Resolución De Tareas Que Implican Demostración En Geometría Del Espacio
}

\author{
Resolution Of Tasks Involving Demonstration In Geometry Of Space
}

\author{
Maritza Luna Valenzuela* \\ Pontifícia Universidade Católica de São Paulo - PUCSP \\ Saddo Ag Almouloud** \\ Pontifícia Universidade Católica de São Paulo - PUCSP \\ Francisco Ugarte Guerra ${ }^{* * *}$ \\ Pontificia Universidad Católica de Perú - PUCP
}

\begin{abstract}
Resumen
En este artículo, estudiamos procesos relacionados a la resolución de tareas que involucran prueba y demostración. El objetivo es identificar el Modelo Epistemológico Dominante (MED) peruano, los tipos de procesos (técnicas) y las herramientas matemáticas (tecnologías) que se utilizan para solucionar una tarea que requiere demostración en geometría del espacio. Para el análisis se empleó la noción de praxeología de la Teoría Antropológica de lo Didáctico (TAD). Los resultados obtenidos fueron detectar las técnicas y las tecnologías para resolver una tarea y además mostrar las articulaciones de las tecnologías, el tipo de organización matemática que permitirá construir una organización didáctica teniendo presente el modelo dominante peruano.
\end{abstract}

Palabras-chave: Prueba y demostración, Baricentro del Tetraedro, Teoría Antropológica de lo Didáctico.

\begin{abstract}
In this article, we study processes related to solving tasks that involve proof and demonstration. The objective is to identify the Peruvian Dominant Epistemological Model (MED), the types of processes (techniques) and mathematical tools (technologies) that are used to solve a task that requires demonstration in space geometry. For the analysis, the notion of praxeology of the Anthropological Theory of the Didactic (TAD) was used. The results obtained were to detect the techniques and technologies to solve a task and show the articulations of the technologies, the type of mathematical organization that will allow to build a didactic organization keeping in mind the dominant Peruvian model.
\end{abstract}

Keywords: Proof and demonstration, Tetrahedron Baricenter, Anthropological Theory of the Didactic.

\footnotetext{
* Mestre en Matemáticas en la Pontificia Universodad Católica del Perú - PUCP. Profesora a tiempo parcial de la Pontificia Universidad Católica de Perú - PUCP. Doutoranda na Pontifica Universidade Católica de São Paulo Brasil (PUC/SP), São Paulo, São Paulo, Brasil. E-mail: luna.m@pucp.edu.pe

** Doctor en Matemáticas y Aplicaciones de la Université de Rennes I de Francia. Doutor asistente en la Pontifícia Universidade Católica de São Paulo - PUC/SP, São Paulo, Brasil. E-mail: saddoag@gmail.com

*** Doctor en Matemáticas por la Universidad de Valladolid (UVa). Profesor principal de la Pontificia Universidad Católica de Perú - PUCP y Director del IREM PUCP, Lima, Perú. E-mail: fugarte@pucp.edu.pe
} 


\section{Introducción}

La utilización de envases en forma de tetraedro revolucionó este concepto, no solo por el material utilizado: papel, plástico y aluminio, sino también por la optimización de material utilizado en su construcción. El nuevo envase fue llamado Tetra Pak, presentado por Ruben Rausing en 1951, está constituido por cuatro caras que son triángulos. Si son triángulos equiláteros, es decir iguales entre sí, se denomina un tetraedro regular, también conocido como uno de los cinco sólidos denominados platónicos.

En este artículo analizaremos una tarea que incluye la resolución de un problema de geometría que requiere demostrar que las bimedianas de un tetraedro son concurrentes, es decir, que se cortan en un punto conocido como baricentro. El análisis de la demostración presentada se realizará a la luz de la Teoría Antropológica de lo Didáctico (TAD) de modo que mostraremos algunas técnicas utilizadas, la tecnología empleada, así como su teoría. Esto nos permitirá no solo identificar los tipos de procesos y las herramientas matemáticas que se utilizan para dar solución a una determinada tarea, sino que permitirá mostrar cómo se articulan las tecnologías y el tipo de organización matemática. Finalmente, a partir de este análisis proponemos una organización didáctica para el estudio de baricentro del tetraedro.

\section{La Enseñanza De La Geometría En La Educación Básica Regular Y Primer Ciclo De La Educación Superior De Perú}

La educación primaria inicia a los 6 años de edad, consta de 6 grados, un grado por año, donde se considera como ciclo III (1ro y 2 do grado), ciclo IV (3ro y 4to grado) y ciclo V (5to y 6to grado). Para luego continuar la educación secundaria que consta de 5 grados, un grado por año, con el ciclo VI (1 ro y 2do grado), ciclo IV (3ro y 4to grado) y ciclo V (5to grado).

Además, el estudio de sólidos geométricos se encuentra presente en el Programa Curricular Nacional de Perú en los niveles primario y secundario del Área curricular de Matemática. Este contenido, se introduce desde el primer grado nivel primario donde el alumno debe resolver problemas de forma, movimiento y localización, combina las siguientes capacidades:

- Modela objetos con formas geométricas y sus transformaciones.

- Comunica su comprensión sobre las formas y relaciones geométricas.

- Usa estrategias y procedimientos para orientarse en el espacio.

- Argumenta afirmaciones sobre relaciones geométricas. 
La descripción del nivel de competencia esperado al final del ciclo III y IV (de primero a sexto grado) considera "Resuelve problemas en los que modela características y datos de ubicación de los objetos a formas bidimensionales y tridimensionales, sus elementos, propiedades, su movimiento y ubicación en el plano cartesiano. Elabora afirmaciones sobre las figuras compuestas; así como relaciones entre una forma tridimensional y su desarrollo en el plano; las explica con ejemplos concretos y gráficos” (Perú, 2018 (1) p. 256). Por ejemplo, al final de cuarto grado se espera que el estudiante podría decir: "Un cubo se puede construir con una plantilla que contenga 6 cuadrados del mismo tamaño".

Para la descripción del nivel de la competencia esperado al final del ciclo V (quinto y sexto grado) indica:

Resuelve problemas en los que modela las características y la ubicación de objetos del entorno a formas bidimensionales y tridimensionales, sus propiedades, su ampliación, reducción o rotación. Describe y clasifica prismas rectos, cuadriláteros, triángulos, círculos, por sus elementos: vértices, lados, caras, ángulos, y por sus propiedades; usando lenguaje geométrico. Realiza giros en cuartos y medias vueltas, traslaciones, ampliación y reducción de formas bidimensionales, en el plano cartesiano. [...] Explica sus afirmaciones sobre relaciones entre elementos de las formas geométricas y sus atributos medibles, con ejemplos concretos y propiedades (Perú, 2018 (1), p. 260).

Es decir, el alumno al final del nivel primario reconoce y distingue formas y gráficas de figuras geométricas en dos y tres dimensiones, por ejemplo, en dos dimensiones se abordan: triangulo, rectángulos y circunferencias y en tres dimensiones identifica caras de sólidos con mayor énfasis en prisma recto y cilindros.

En la secundaria son retomados los sólidos geométricos tanto irregulares como regulares para trabajar con la geometría sintética y así hallar la medida de las aristas, apotemas de las caras, áreas, y volúmenes.

La descripción del nivel de la competencia esperado al final del ciclo VI (primero y segundo grado de secundaria) menciona:

Resuelve problemas en los que modela características de objetos mediante prismas, pirámides y polígonos, sus elementos y propiedades, y la semejanza y congruencia de formas geométricas; así como la ubicación y movimiento mediante coordenadas en el plano cartesiano, mapas y planos a escala, y transformaciones. Expresa su comprensión de las formas congruentes y semejantes, la relación entre una forma geométrica y sus diferentes perspectivas; usando dibujos y construcciones. Clasifica prismas, pirámides y polígonos, según sus propiedades. Selecciona y emplea estrategias, procedimientos y recursos para determinar la longitud, área o volumen de formas geométricas en unidades convencionales y para construir formas geométricas a escala. Plantea afirmaciones sobre la semejanza y congruencia de formas, relaciones entre áreas de formas geométricas; las justifica mediante ejemplos y propiedades geométricas (Perú, 2018 (2), p. 165).

En el nivel superior podemos observar que parte de la sumilla del curso de Álgebra 


\section{Matricial y Geometría Analítica presenta:}

(...) se abarca los siguientes tres temas: geometría analítica, con las definiciones y propiedades importantes de la recta, circunferencia, parábola, elipse e hipérbola, y la rotación de ejes; álgebra matricial, que comprende las definiciones y operaciones de vectores y matrices aplicados en la resolución de sistemas lineales homogéneos y no homogéneos, y que, además, incluye el cálculo de vectores y valores propios de una matriz; números complejos y sus operaciones básicas. En este curso, se propone la aplicación de todos estos temas en la resolución de problemas intramatemáticos y extramatemáticos. (Sumilla de Álgebra Matricial y Geometría Analítica, 2018).

A continuación, en la figura 1 se muestra el proceso mediante el cual se estudia figuras en el plano y el espacio, en el nivel básico regular, es decir nivel primario y secundario, y el nivel superior.

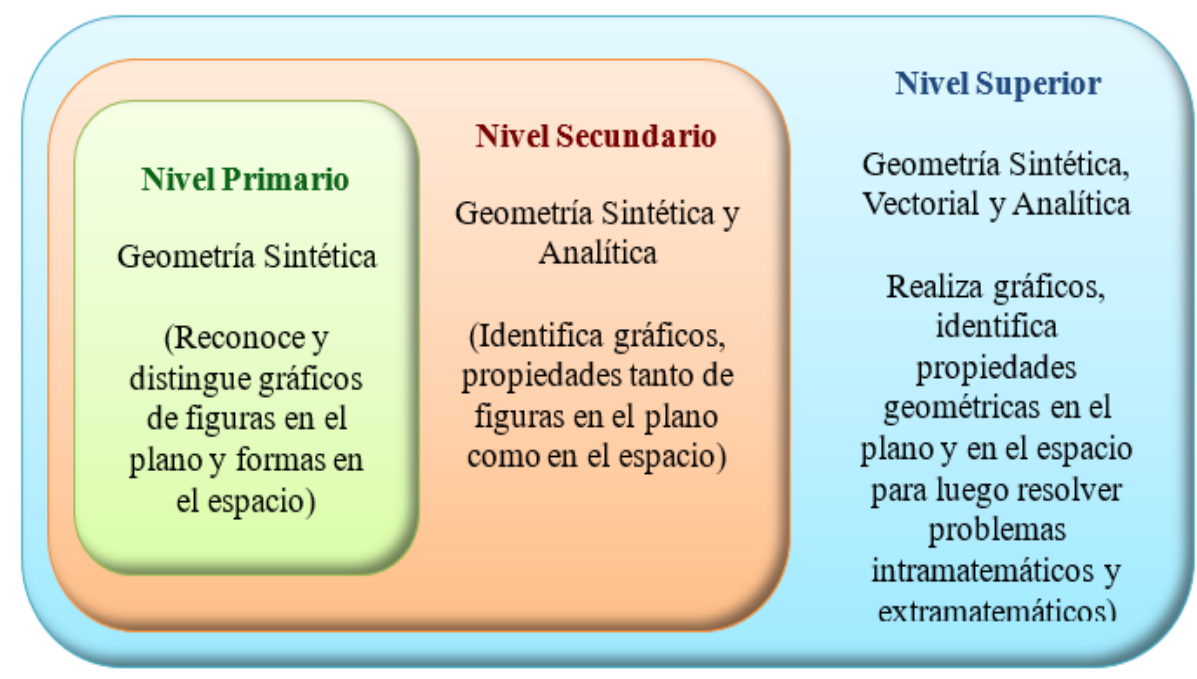

Figura 1. Evolución del estudio de sólidos geométricos Fuente. Los autores

\section{Fundamentación Teórica}

La TAD permite realizar el análisis de las distintas maneras de dar solución a una tarea, ya que según Chevallard: "toda actividad humana regularmente realizada puede describirse con un modelo único, que se resume aquí con la palabra de praxeología” (1999, p. 2). Es decir, el nivel praxis o "saber-hacer" engloba un tipo de tareas y preguntas, así como las técnicas empleadas para llevarlas a cabo. El nivel logos o "saber", en el que los discursos describen, explican y justifican las tareas y técnicas, es llamado tecnología de la técnica. Toda tecnología necesita también de una justificación a lo que se denomina teoría de la tecnología.

Chevallard (1999) define:

Tipos de Tareas $(T)$ : como un conjunto de tareas $(t)$, donde una tarea $(t \in T)$ es una acción en un objeto específico que es ejecutado por alguien, definido por un verbo, llamado género de 
tarea. Además, el género de tarea es la acción que agrupa los tipos de tareas.

Técnica $(\tau)$ : es la manera de resolver una tarea específica.

Tecnología $(\theta)$ : es un discurso racional, cuya primera función es justificar la técnica $\tau$, para ejecutar un tipo de tarea. Una segunda función de la tecnología es explicar la técnica para que ella sea inteligible. La última función de la tecnología es la producción de nuevas técnicas más eficientes y adaptadas para la resolución de una tarea específica.

Teoría $(\Theta)$ : es el discurso racional sobre la tecnología, esto es, aquella que justifica y explica las afirmaciones de la tecnología.

Se puede concluir que alrededor de una tarea $(T)$, se encuentra una técnica $(\tau)$ sustentada por una tecnología $(\theta)$, que a su vez es explicada por una teoría $(\Theta)$. Finalmente $[T / \tau / \theta / \Theta]$ constituye una praxeología puntual, relativa a un único tipo de tarea. Es decir, una praxeología está constituida por un bloque práctico-técnico, $[\mathrm{T} / \tau]$, y por un bloque tecnológico-teórico, $[\theta / \Theta]$.

Se tiene presente que las praxeologías integradas a un saber matemático son la organización matemática (OM) y la organización didáctica (OD). La OM estudia la situación identificada en las tareas, técnicas, tecnología y teoría. La OD observa la manera como estas situaciones fueron constituidas, por intermedio de momentos de estudio. La noción de "momento" fue concebida por Chevallard (1999) para delinear una OD y está estructurada en seis etapas.

Las praxeologías, de acuerdo con el grado de complejidad de sus componentes, se clasifican de la siguiente manera: Organización Matemática Puntual (OMP), considerada apenas un tipo de tarea; Organización Matemática Local (OML), que deriva de la integración de varias praxeologías puntuales que atienden a una misma tecnología; Organización Matemática Regional (OMR), obtenida de la articulación de praxeologías locales referentes a la misma teoría matemática; y Organización Matemática Global (OMG), que surge de la unión de diferentes praxeologías regionales, a partir de la integración de diversas teorías.

Siguiendo el constructo de investigación propuesto por la TAD (Chevallard, 1999), para abordar a dimensión epistemológica de la construcción del problema didáctico, precisamos de un Modelo Epistemológico de Referencia (MER) sobre vectores. Por esa razón, el MER constituye un instrumento de emancipación del investigador y de la didáctica, ya que permite cuestionar la manera como las instituciones envueltas en la problemática didáctica interpretan el conocimiento matemático.

El MER también es esencial para estudiar el conocimiento matemático antes de ser 
transformado en objeto a ser enseñado. Es el instrumento con el cual el investigador puede desconstruir y reconstruir las praxeologías cuya divulgación intrainstitucional e interinstitucional pretende analizar.

Un MER depende de la intención de quien lo propone, sirve para cuestionar y analizar los modelos epistemológicos denominados dominantes (Gascón, 2003; 2011) Así, un modelo de referencia que predomina en una institución es denominado modelo de referencia_dominante (MED).

En la perspectiva de la Didáctica de la Matemática hay necesidad de tener un entendimiento mínimo sobre uno o más objetos matemáticos, lo que implica que hay necesidad de tener presente un MER para contrastar el Modelo Epistemológico Dominante (MED) y concebir una organización didáctica matemática (OMD) fundamentada en la razón de ser de los objetos en estudio y de su articulación en nivel de complejidad creciente.

También se tendrá presente el concepto de prueba y demostración definida por Balacheff (1987), es decir prueba es una explicación planteada por una comunidad. Mientras que demostración es una prueba realizada por matemáticos respetando ciertos criterios rigurosos de reglas o axiomas.

\section{Metodología}

Esta investigación sigue los procesos de las contribuciones de la TAD para determinar y analizar qué técnicas, tecnologías y teorías se aplican para realizar una tarea concreta, en el contexto de la geometría. Dicho análisis, nos permitirá identificar una organización matemática, reconocer su tipo y analizarla con miras a plantear, más adelante, un modelo epistemológico de referencia alternativo y una organización didáctica para la enseñanza de las demostraciones en geometría en el espacio.

\section{Procesos}

Para lograr nuestro propósito se exhibirán cinco formas de realizar demostraciones sobre la intersección de bimedianas de un tetraedro. Asimismo, en el análisis se explicará cada una de las formas en las fue resuelta la tarea (técnicas), las propiedades (tecnologías) y contenidos matemáticos utilizados (teoría). Se finalizará con el análisis a partir de la esquematización de las comparaciones y articulaciones realizadas por las demostraciones. 


\section{Análisis de los resultados}

La tarea solicitada es la siguiente:

Determinar el centro de gravedad o baricentro de un tetraedro regular.

Dicho de otra manera, se puede enunciar la tarea del siguiente modo:

Demostrar que el centro de gravedad o baricentro de un tetraedro regular es igual al punto de concurrencia de las bimedianas.

Además, debemos tener presente que en un tetraedro regular las cuatro caras son triángulos equiláteros y presentan sus respectivos baricentros, que este caso son también incentro, ortocentro y circuncentro. Para de determinar el baricentro del tetraedro se necesita trazar las bimedianas, es decir, son rectas que unen el baricentro de cada triángulo (cara de tetraedro) con el vértice que se le opone respectivamente. De la intersección de dos de dichas bimedianas se obtiene el baricentro.

Para dar solución a la tarea, a continuación, se describen cinco procedimientos o técnicas que incluyen propiedades o tecnologías de la Geometría Sintética, de la Geometría Analítica y de la Geometría Vectorial.

Para abordar la primera forma de solución (técnica) se utilizarán herramientas (tecnologías) de la geometría sintética, también conocida como la geometría euclidiana, teoría que se ocupa del estudio de figuras en el plano y en el espacio (puntos, líneas rectas, círculos, triángulos, etc.), donde los teoremas se demuestran deduciéndolos a partir de los axiomas y postulados de Euclides.

\section{Solución (forma 1).}

Para poder dar solución a la tarea, debemos determinar la razón a la que divide el baricentro del tetraedro a cada bimediana.

Para tal efecto, consideramos un tetraedro de vértices $V A B C$ y arista $a$. Además, en el triángulo $\triangle A B C$ tenemos que si $F$ es el baricentro de dicho triángulo, entonces este punto divide a cada mediana en una razón de 2 a 1 . De modo que, si la arista del tetraedro mide $a$, por ende, el lado del triángulo mide $a$, lo cual implica que la mediana mide $\frac{\sqrt{3}}{2} a$ en particular $C M_{2}$ y $V M_{2}$. Por lo tanto, $\frac{M_{2} F}{F C}=\frac{1}{2}$ o que $C F=\frac{2\left(\frac{\sqrt{3}}{2} a\right)}{3}=\frac{\sqrt{3}}{3} a$.

Así, CF mide $\frac{\sqrt{3}}{3} a$, como se muestra detalles en la figura 2 . 


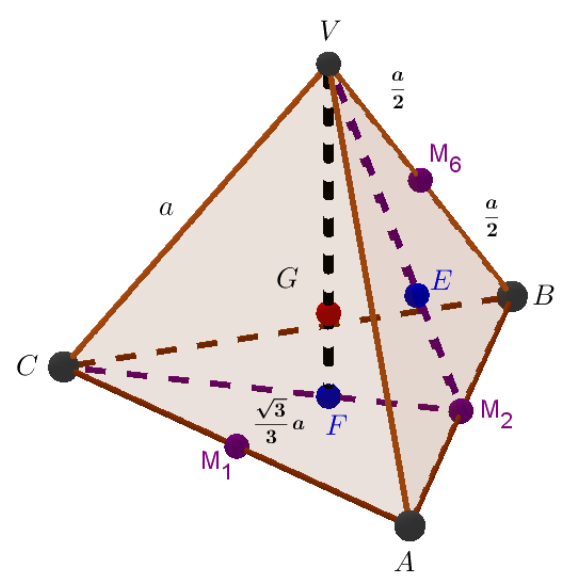

Figura 2. Baricentro del tetraedro. Fuente. Los autores.

Además, consideramos el baricentro $E$ del triángulo $\triangle V A B$ para poder trazar su bimediana, en este caso consideramos la bimediatriz a la recta que pasa por el vértice $C$ y el punto $\mathrm{E}$, denotada por $L_{C E} \mathrm{y}$ otra bimediana a la recta que pasa por el vértice $V$ y el punto $\mathrm{F}$, denotada por $L_{V F}$. De modo que $L_{C E} \cap L_{V F}=\{G\}$, es decir, de la intersección de las rectas tenemos el baricentro $G$.

Seguidamente, debemos encontrar la longitud de $V F$, para lo cual consideramos el triángulo rectángulo $\triangle V F C$, recto en $\mathrm{F}$, como se puede apreciar en la figura 3 . Aplicando el teorema de Pitágoras tenemos

$$
V F=\sqrt{a^{2}-\left(a \frac{\sqrt{3}}{3}\right)^{2}}=\frac{\sqrt{6}}{3} a .
$$

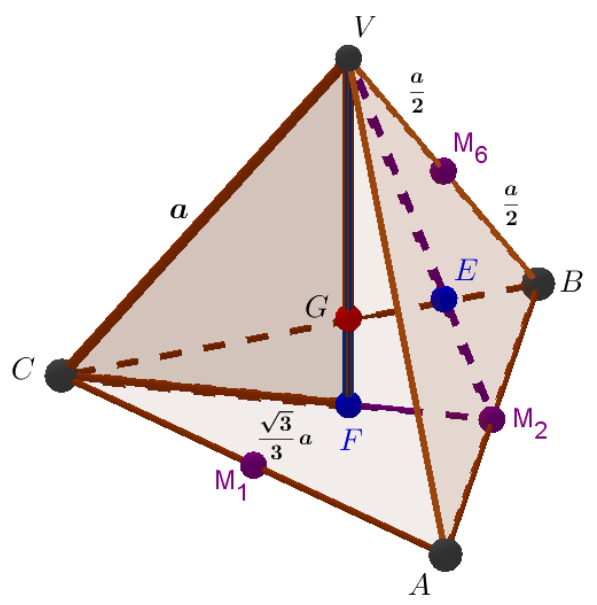

Figura 3. Longitud de la altura de un tetraedro regular Fuente: Los autores.

Ahora en el triángulo $\Delta V M_{2} C$ tenemos que $V E$ tiene la misma medida que $C F$ e igual a 
$\frac{\sqrt{3}}{3} a$ y también $E M_{2}$ tiene la misma medida igual a $F M_{2}$ e igual $\frac{\sqrt{3}}{6} a$.

Recordemos que las bimedianas $V F$ y $C E$ tienen medida igual a $\frac{\sqrt{6}}{3} a$. Podemos notar que $E M_{2}$ mide igual a $M_{2} F$ y $G M_{2}$ es un lado en común para los triángulos $\Delta M_{2} F G$ y $\Delta M_{2} E G$ por criterio de congruencia LAL, entonces se tiene que los triángulos $\Delta M_{2} F G$, y $\Delta M_{2} E G$ son congruentes. Lo cual conduce a que el lado $E G$ mida igual al lado $F G$. Ello implica que $C G$ mida igual a $V G$, ver detalles en la figura 4.

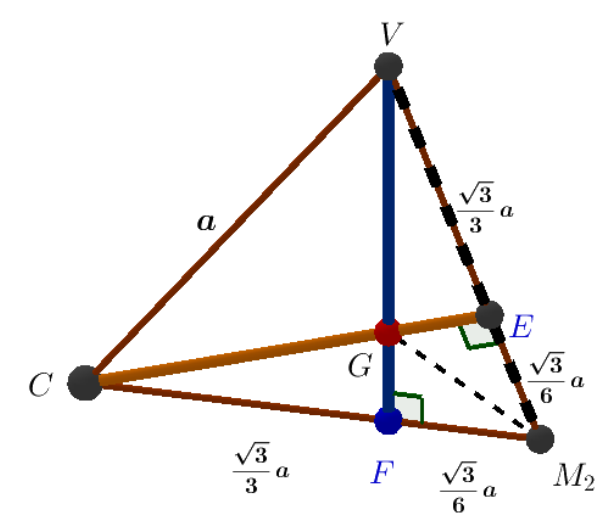

Figura 4. Congruencia de triángulos $\Delta M_{2} F G$ y $\Delta M_{2} E G$.

Fuente: Los autores.

Seguidamente observamos la bimediana $V F=V G+G F$ o $\frac{\sqrt{6}}{3} a=V G+G F$, y también $(G F)^{2}=(C G)^{2}-(C F)^{2}$ о $(G F)^{2}=(C G)^{2}-\left(\frac{\sqrt{3}}{3} a\right)^{2}$ y como $V G=C G$,

entonces resolvemos las ecuaciones

$$
\begin{aligned}
& (G F)^{2}=(V G)^{2}-\left(\frac{\sqrt{3}}{3} a\right)^{2} \\
& \frac{\sqrt{6}}{3} a=V G+G F .
\end{aligned}
$$

Tenemos $V G=\frac{\sqrt{6}}{4} a$ y $G F=\frac{\sqrt{6}}{12} a$. Se puede ver detalles en la figura 5. Así, la razón a la que divide $\mathrm{G}$ a la bimediana esta en razón de

$$
\frac{V G}{G F}=\frac{\frac{\sqrt{6}}{4} a}{\frac{\sqrt{6}}{12} a}=\frac{3}{1} .
$$




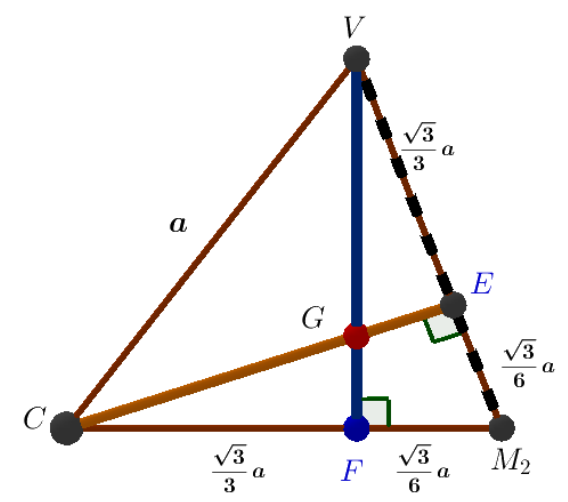

Figura 5. Razón 3 a 1 que divide el baricentro $G$ a la bimediana $V F$. Fuente: Los autores.

La siguiente demostración será realizada en la geometría vectorial (teoría), es decir, en la geometría que se ocupa del estudio de vectores (definido por una dirección, sentido y una longitud). La introducción de esta herramienta matemática hace posible, en particular, controlar la alineación de los puntos, el paralelismo o la perpendicularidad de las líneas, determinar las coordenadas de puntos particulares, de la distancia entre dos puntos, calcular ángulos, realizar operaciones algebraicas (tecnologías).

Además se considerará la razón de 1 a 3 divide en baricentro de una bimediana (técnica que consideran en Collectión Inter Africaine de Mathematique). Por lo tanto, se deberá demostrar que las 4 bimedianas coinciden en un mismo punto $\mathrm{G}$.

\section{Solución (forma 2)}

Dado los cuatro puntos de los vértices del tetraedro, A, B, C y V. Sean E, F, H y K los baricentros de las caras $\triangle V A B, \triangle A B C, \triangle V A C$ y $\triangle V B C$ respectivamente, como podemos apreciar en la figura 6.

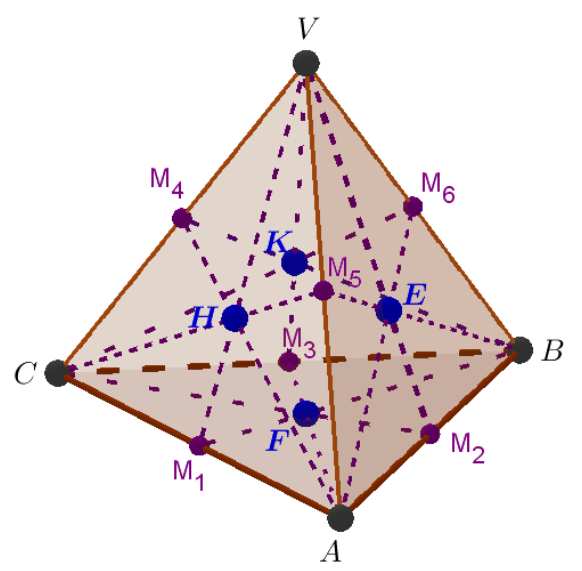

Figura 6. Baricentros de las caras del tetraedro Fuente: Los autores. 
Consideran que $O$ es el origen de coordenadas, entonces tenemos

$$
\begin{array}{ll}
E-O=\frac{(V-O)+(A-O)+(B-O)}{3} & F-O=\frac{(A-O)+(B-O)+(C-O)}{3} \\
H-O=\frac{(V-O)+(A-O)+(C-O)}{3} & K-O=\frac{(V-O)+(B-O)+(C-O)}{3}
\end{array}
$$

Calculando la bimediana que une los puntos $A$ y $K$ tenemos $G_{1}$

$$
\begin{gathered}
G_{1}-O=\frac{(A-O)+3(K-O)}{1+3} \\
G_{1}-O=\frac{(A-O)+3 \frac{(V-O)+(B-O)+(C-O)}{3}}{1+3} \\
G_{1}-O=\frac{(A-O)+(V-O)+(B-O)+(C-O)}{4} .
\end{gathered}
$$

Similarmente hallamos $G_{2}$, considerando $B$ y $H$,

$$
\begin{gathered}
G_{2}-O=\frac{(B-O)+3(H-O)}{1+3} \\
G_{2}-O=\frac{(B-O)+(V-O)+(A-O)+(C-O)}{4} .
\end{gathered}
$$

Luego $G_{3}$, teniendo en cuenta $C$ y $E$,

$$
\begin{gathered}
G_{3}-O=\frac{(C-O)+3(E-O)}{1+3} \\
G_{3}-O=\frac{(C-O)+(V-O)+(A-O)+(B-O)}{4} .
\end{gathered}
$$

Finalmente $G_{4}$, a partir del vértice $V$ y el baricentro $F$,

$$
\begin{gathered}
G_{4}-O=\frac{(V-O)+3(F-O)}{1+3} \\
G_{4}-O=\frac{(V-O)+(A-O)+(B-O)+(C-O)}{4} .
\end{gathered}
$$

Del calculo realizado, podemos observar que los cuatro baricentros exactamente identicos, $G_{1}=G_{2}=G_{3}=G_{4}=G$. Como se muestra en la figura 7. Por tanto el punto $G$ es obtenido a partir de la cuarta parte de la suma de los cuatro vértices, es decir

$$
G=\frac{A+B+C+D}{4}
$$




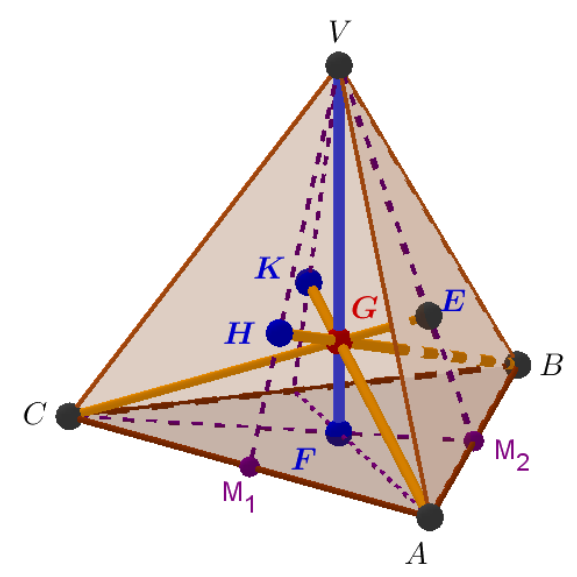

Figura 7. Intersección de bimedianas Fuente: Los autores.

La siguiente forma de solución es realizada considerando la geometría vectorial (sin coordenadas).

Teniendo en cuenta que la geometría vectorial se tiene elementos denominados vectores que deben tener en cuenta tres caracteristicas: dirección, sentido y longitud.

\section{Solución (forma 3)}

Dado los cuatro puntos de los vértices del tetraedro, A, B, C y V. Se define la bimediana como el segmento que es trazado a partir de un vétice al baricentro de su cara opuesta.

Para el vértice $C$ se traza al baricentro de su cara opuesta $\triangle A V B$, es decir $E=\frac{A+B+V}{3}, \mathrm{y}$ para el vétice $V$ se traza al baricentro de su cara opuesta $F=\frac{A+B+C}{3}$ teniendo las cuatro medianas, una para cada vértice. Determinaremos que las medianas se intersecan en el punto $G$, como se muestra en la figura 8 .

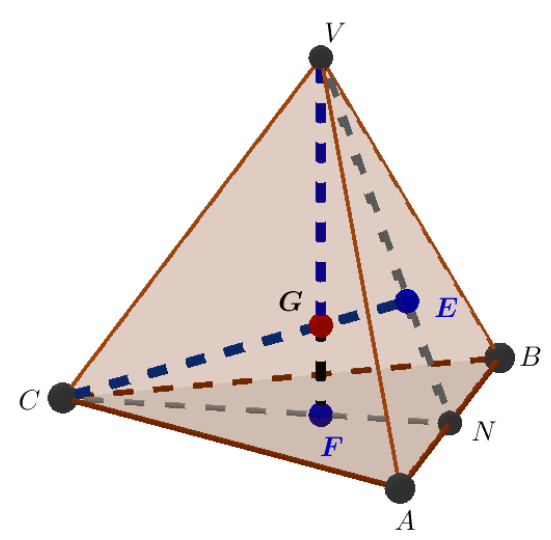

Figura 8 Baricentro del tetraedro. Fuente: Los autores. 
Teniendo las ecuaciones:

$$
\begin{cases}G=\alpha C+(1-\alpha) \frac{A+B+V}{3}, & \alpha \in[0,1] \\ G=\beta V+(1-\beta) \frac{A+B+C}{3}, & \beta \in[0,1]\end{cases}
$$

Igualando dichas ecuaciones tenemos:

$$
\alpha C+(1-\alpha) \frac{A+B+V}{3}=\beta V+(1-\beta) \frac{A+B+C}{3} .
$$

De donde

$$
\begin{gathered}
A\left(\frac{(1-\alpha)}{3}-\frac{(1-\beta)}{3}\right)+B\left(\frac{(1-\alpha)}{3}-\frac{(1-\beta)}{3}\right)+C\left(\alpha-\frac{(1-\beta)}{3}\right)+V\left(\frac{(1-\alpha)}{3}-\beta\right) \\
=0 \\
A\left(\frac{\beta-\alpha}{3}\right)+B\left(\frac{\beta-\alpha}{3}\right)+C\left(\alpha-\frac{1-\beta}{3}\right)=-V\left(\frac{1-\alpha}{3}-\beta\right) .
\end{gathered}
$$

Se tiene que $\mathrm{V}$ es un punto que esta en el espacio que no puede ser escrito como combinación lineal de los puntos A, B y C que estan en un mismo plano. Por tanto,

$$
\frac{1-\alpha}{3}-\beta=0 \quad \text { y } A\left(\frac{\beta-\alpha}{3}\right)+B\left(\frac{\beta-\alpha}{3}\right)+C\left(\alpha-\frac{1-\beta}{3}\right)=0 .
$$

De la segunda igualdad tenemos:

$$
(A+B)\left(\frac{\beta-\alpha}{3}\right)=C\left(\frac{1-\beta}{3}-\alpha\right)
$$

Como $\overrightarrow{O A}+\overrightarrow{O B}$ y $\overrightarrow{O C}$ son coplanares no paralelos, la igualdad es verdad si

$$
\frac{\beta-\alpha}{3}=0 \text { y } \frac{1-\beta}{3}-\alpha=0 \text {. }
$$

Por lo tanto $\beta=\alpha=\frac{1}{4}$, de donde se tiene que la ecuación del baricentro es:

$$
G=\frac{A+B+C+D}{4}
$$

Lo que concluye la demostración.

La siguiente manera de resolver en la geometría vectorial y analítica (con coordenadas), es decir considerando la ecuación vectorial de la recta.

\section{Solución (forma 4)}

Considerando las rectas bimedianas, una que pasan por los puntos $\mathrm{C}$ y $\mathrm{E}$, denotada por $L_{C E}$ y otra que pasas por $V$ y F, $L_{V F}$, escritas en forma paramétrica, y se representa como el conjunto de puntos donde cada uno tiene la forma del punto P. Asi, la recta puede ser escrita vectorialmente como:

$$
L_{C E}: P=C+t(\overrightarrow{E C}), \text { con } t \in \mathbb{R}
$$




$$
L_{V F}: P=V+s(\overrightarrow{V F}), \text { con } s \in \mathbb{R} .
$$

Entonces de la intersección de estas dos rectas tenemos el punto $G$, es decir

$$
L_{C E} \cap L_{V F}=\{G\} .
$$

En el proceso de resolución se tiene la ecuación

$$
C+t(\overrightarrow{E C})=V+s(\overrightarrow{V F}), \text { con } t, s \in \mathbb{R} .
$$

O dicho de otro modo

$$
\begin{gathered}
\left(x_{C}, y_{C}, z_{C}\right)+t\left(x_{E C}, y_{E C}, z_{E C}\right)=\left(x_{V}, y_{V}, z_{V}\right)+s\left(x_{V F}, y_{V F}, z_{V F}\right) \\
\left\{\begin{array}{l}
x_{C}+t x_{E C}=x_{V}+s x_{V F} \\
y_{C}+t y_{E C}=y_{V}+s y_{V F} \\
z_{C}+t z_{E C}=z_{V}+s z_{V F} .
\end{array}\right.
\end{gathered}
$$

En el sistema podemos observar que se tiene tres ecuaciónes lineales y además se tiene dos variables $t$ y $s$, es decir el número de variables es menor al numero de ecuaciones (bastaria tener en cuenta dos ecuación y los resultados deben satisfacer la tercera ecuación), entonces el sistema tiene una solución única. Luego, de la solución del sistema de ecuaciones se tiene las coordenadas explicitas del baricentro $G$. Podemos ver la representación gráfica de dos rectas (en este caso solo se graficaron dos rectas) en la figura 9. Del mismo modo se procede con otro par de rectas para luego encontrar las coordenadas del baricentro.

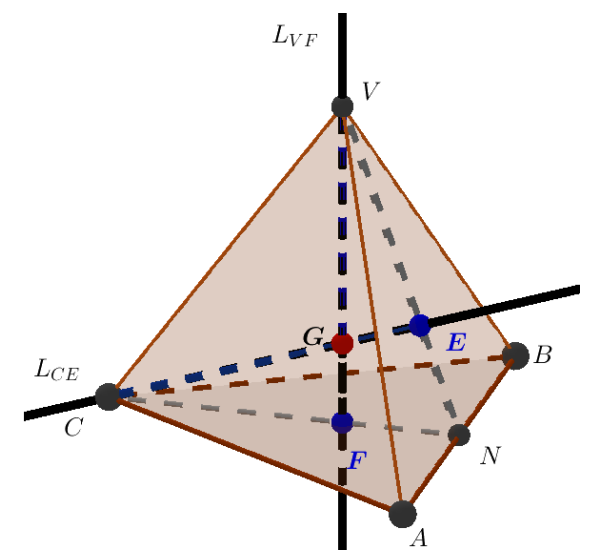

Figura 9. Baricentro del tetraedro como intersección de rectas.

Fuente: Los autores.

La siguiente forma de solución es realizada en la Geometría Analítica y la Geometría Vectorial, considerando planos que pasan por el punto medio y baricentro de cada cara del tetraedro. Son cuatro planos, pero bastará considerar tres de ellos para encontrar el Baricentro que buscamos, ya que se procede de manera similar si se considera otra combinación de otros tres planos.

\section{Solución (forma 5)}

Para determinar el baricentro del tetraedro considerando los planos $\pi_{V C M_{2}}, \pi_{V B M_{1}} \mathrm{y}$ 
$\pi_{C B M_{5}}$, de la intersección de dichos planos se obtiene el punto $\mathrm{G}$.

Además se puede realizar traslaciones y rotaciones de modo que el tetraedro se puede ubicar en el espacio de diversas formas.

Sin perdida de generalidad consideramos la cara $A B C$ del tetraedro contenida en el plano $X Y$, con el baricentro de dicha cara en el origen de coordenadas, de manera que los vértices $A, B, C$ tienen la tercera coordenada igual a cero, además el vértice $A$ ubicamos en el eje $Y$ con $y_{A}>0$, es decir $A\left(0, y_{A}, 0\right)$. Por lo tanto, los vértices $B\left(x_{B}, y_{B}, 0\right)$ y $C\left(x_{C}, y_{C}, 0\right)$ serían simétricos respecto al eje $Y$, con la condición que $x_{B}=-x_{C}, y_{B}=y_{C}, \mathrm{y} y_{C}<0$. El vértice $V$ de coordenadas $\left(0,0, z_{V}\right)$. Como se puede visualizar en a figura 10 .

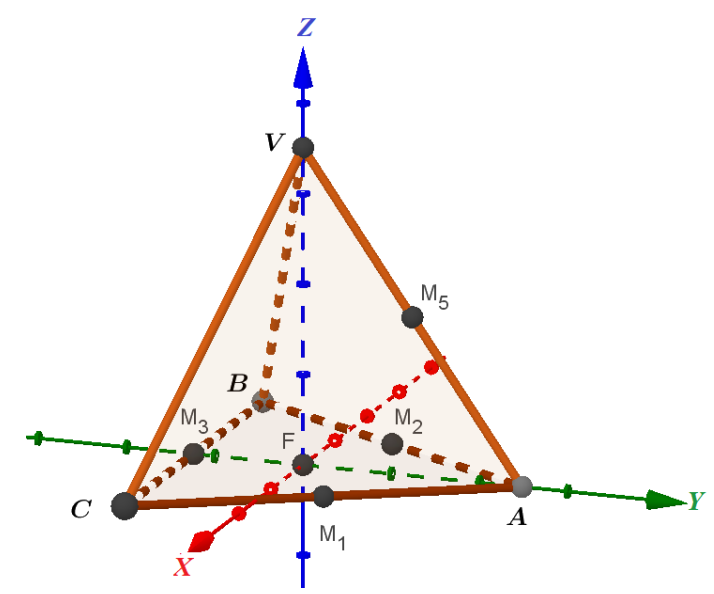

Figura 10. Tetraedro en el sistema de coordenadas $X Y Z$ Fuente: Los autores.

Para cada plano $\pi_{V C M_{2}}, \pi_{V B M_{1}}$ y $\pi_{C B M_{5}}$, se tiene la siguiente ecuación:

$$
\begin{gathered}
\pi_{V C M_{2}}:(P-V) \cdot(\overrightarrow{A B})=0 \\
\left((x, y, z)-\left(0,0, z_{V}\right)\right) \cdot\left(x_{B}, y_{B}-y_{A}, 0\right)=0 \\
\left(x, y, z-z_{V}\right) \cdot\left(x_{B}, y_{B}-y_{A}, 0\right)=0 \\
x_{B} x+\left(y_{B}-y_{A}\right) y+0\left(z-z_{V}\right)=0 \\
x_{B} x+\left(y_{B}-y_{A}\right) y+0 z=0 .
\end{gathered}
$$

Para el plano $\pi_{V B M_{1}}$ tenemos

$$
\begin{aligned}
& \pi_{V B M_{1}}:(P-V) \cdot(\overrightarrow{A C})=0 \\
& x_{C} x+\left(y_{C}-y_{A}\right) y+0 z=0
\end{aligned}
$$

De modo similar tenemos $\pi_{C B M_{5}}$

$$
\begin{aligned}
& \pi_{C B M_{5}}:(P-B) \cdot(\overrightarrow{A V})=0 \\
& 0 x-y_{A} y+\left(z_{V}\right) z=-y_{A} y_{B}
\end{aligned}
$$


Así, el sistema que se debe resolver para tener el baricentro es el siguiente:

$$
\left\{\begin{array}{c}
x_{B} x+\left(y_{B}-y_{A}\right) y+0 z=0 \\
x_{C} x+\left(y_{C}-y_{A}\right) y+0 z=0 \\
0 x-y_{A} y+z_{V} z=-y_{A} y_{B}
\end{array}\right.
$$

La solución de sistema es

$$
\left[\begin{array}{ccc}
x_{B} & \left(y_{B}-y_{A}\right) & 0 \\
x_{C} & \left(y_{C}-y_{A}\right) & 0 \\
0 & \left(-y_{A}\right) & \left(z_{V}\right)
\end{array}\right]\left[\begin{array}{l}
x \\
y \\
Z
\end{array}\right]=\left[\begin{array}{c}
0 \\
0 \\
-y_{A} y_{B}
\end{array}\right]
$$

El sistema tendrá solución si

$$
\operatorname{det}\left[\begin{array}{ccc}
x_{B} & \left(y_{B}-y_{A}\right) & 0 \\
x_{C} & \left(y_{C}-y_{A}\right) & 0 \\
0 & \left(-y_{A}\right) & \left(z_{V}\right)
\end{array}\right]=z_{V}\left[x_{B}\left(y_{C}-y_{A}\right)-x_{C}\left(y_{B}-y_{A}\right)\right]
$$

Como $x_{B}=-x_{C}$ y $y_{B}=y_{C}$, tenemos:

$$
\begin{gathered}
=z_{V}\left[x_{B}\left(y_{C}-y_{A}\right)+\left(-x_{C}\right)\left(y_{B}-y_{A}\right)\right]=z_{V}\left[x_{B}\left(y_{C}-y_{A}\right)+x_{B}\left(y_{C}-y_{A}\right)\right] \\
=z_{V} x_{B}\left[\left(2 y_{C}-2 y_{A}\right)\right]=z_{V} x_{B}\left[\left(2 y_{C}-2 y_{A}\right)\right]=2 z_{V} x_{B}\left(y_{C}-y_{A}\right) .
\end{gathered}
$$

Se sabe además que $y_{C}<0$ y $y_{A}>0$ entonces $-y_{A}<0$ y $y_{C}-y_{A}>0$.

$$
\text { Por lo tanto, } \operatorname{det}\left[\begin{array}{ccc}
x_{B} & \left(y_{B}-y_{A}\right) & 0 \\
x_{C} & \left(y_{C}-y_{A}\right) & 0 \\
0 & \left(-y_{A}\right) & \left(z_{V}\right)
\end{array}\right]=2 z_{V} x_{B}\left(y_{C}-y_{A}\right) \neq 0 \text {. }
$$

Por la regla de Cramer (el sistema puede ser resuelto, también, por otros métodos) tenemos

$$
\begin{gathered}
x=\frac{\operatorname{det}\left[\begin{array}{ccc}
0 & \left(y_{B}-y_{A}\right) & 0 \\
0 & \left(y_{C}-y_{A}\right) & 0 \\
-y_{A} y_{B} & \left(-y_{A}\right) & \left(z_{V}\right)
\end{array}\right]}{\operatorname{det}\left[\begin{array}{ccc}
x_{B} & \left(y_{B}-y_{A}\right) & 0 \\
x_{C} & \left(y_{C}-y_{A}\right) & 0 \\
0 & \left(-y_{A}\right) & \left(z_{V}\right)
\end{array}\right]}=0, \quad y=\frac{\operatorname{det}\left[\begin{array}{ccc}
x_{B} & 0 & 0 \\
x_{C} & 0 & 0 \\
0 & -y_{A} y_{B} & \left(z_{V}\right)
\end{array}\right]}{\operatorname{det}\left[\begin{array}{ccc}
x_{B} & \left(y_{B}-y_{A}\right) & 0 \\
x_{C} & \left(y_{C}-y_{A}\right) & 0 \\
0 & \left(-y_{A}\right) & \left(z_{V}\right)
\end{array}\right]}=0, \\
z=\frac{\operatorname{det}\left[\begin{array}{ccc}
x_{B} & \left(y_{B}-y_{A}\right) & 0 \\
x_{C} & \left(y_{C}-y_{A}\right) & 0 \\
0 & -y_{A} & -y_{A} y_{B}
\end{array}\right]}{\operatorname{det}\left[\begin{array}{ccc}
x_{B} & \left(y_{B}-y_{A}\right) & 0 \\
x_{C} & \left(y_{C}-y_{A}\right) & 0 \\
0 & \left(-y_{A}\right) & \left(z_{V}\right)
\end{array}\right]}=-\frac{y_{A} y_{B}\left[x_{B}\left(y_{C}-y_{A}\right)-x_{C}\left(y_{B}-y_{A}\right)\right]}{2 z_{V} x_{B}\left(y_{C}-y_{A}\right)}=-\frac{y_{A} y_{B}}{z_{V}}
\end{gathered}
$$

Así el baricentro $G$ del tetraedro tiene como coordenadas:

$$
G=\left(0,0,-\frac{y_{A} y_{B}}{z_{V}}\right)
$$

Como se muestra en la figura 11. 


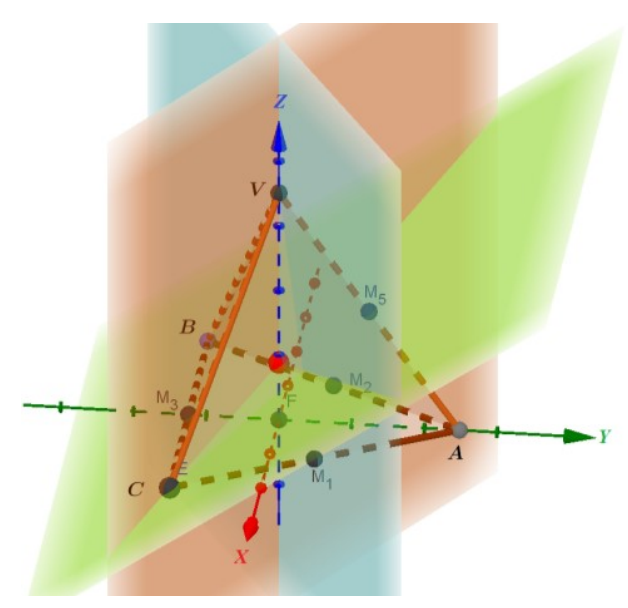

Figura 11. Baricentro del tetraedro como intersección de planos. Fuente: Los autores.

Para la solución de la tarea, con base en la TAD, podemos identificar que se procedio por cinco técnicas. En cada técnica inicia recurriedo al concepto de baricentro de un triángulo; es decir, considera en la geometria euclidiana (sin coordenadas) que el baricentro divide a la mediana (del vértice al punto medio del lado que se le opone) en una razón de 2 a 1 y en la geometría analítica o vectorial se considerá que el baricentro del triángulo es un tercio de la suma de las coordeadas de los vértices de dicho triángulo. Lo cual se debe realizar en cada cara del tetraedro, para luego preceder a determinar las bimedianas, es decir determinar el segmento que une un vértice y el baricentro de la cara del tetraedro que se le opone.

La primera forma para demostrar que el punto $G$ divide al segmento bimediana en una razón de 1 a 3, se aplica división de un segemento por una razón dada, luego congruencia de tríangulos y un par de veces el teorema de Pitagoras, en la Geometría Sintética. Después, se demuestra que el punto $\mathrm{G}$ es donde coincide la intersección de las bimedianas.

La segunda forma, se considera resutados de la primera forma, es decir la división de un segmento bimediano por una razón 3 a 1, para luego determinar las coordenadas de baricentro del teraedro que son la cuarta parte de la suma de los cuatro vértices del tetraedro.

En la tercera forma de demostración es concebida en la Geometria Vectorial utiliza las propiedades de vectores tales como la igualdade, la adición y el paralelismo de vectores, para así poder determinar el baricentro.

Para cuarta manera de demostrar se hizo en la Geometría Analítica y Vectorial puesto que se usa la definición de una recta que es un punto paso más el producto de un parametro y un vector dirección, luego se procede a resolver el sistema de ecuaciones lineales. Así, se logra determinar las coordenadas de baricentro.

De las técnicas empleadas para realizar la demostración, podemos resaltar los teoremas 
y propiedades utilizadas en el cuadro 1 mostrado a continuación.

Cuadro 1. Técnicas para resolver tarea.

\begin{tabular}{|c|c|c|c|c|}
\hline \multicolumn{5}{|c|}{ Modelos de demonstración } \\
\hline Geometría Sintética & \multicolumn{2}{|c|}{$\begin{array}{l}\text { Geometría Vectorial } \\
\text { (Sin coordenada) }\end{array}$} & \multicolumn{2}{|c|}{$\begin{array}{l}\text { Geometria Analítica y Vectorial } \\
\text { (Con coordenada) }\end{array}$} \\
\hline$\left(\tau_{1}\right)$ & $\left(\tau_{2}\right)$ & $\left(\tau_{3}\right)$ & $\left(\tau_{4}\right)$ & $\left(\tau_{5}\right)$ \\
\hline $\begin{array}{l}\text { Baricentro de un } \\
\text { triángulo } \\
\text { Teorema de Pitágoras } \\
\text { Congruencia de } \\
\text { triángulos } \\
\quad \text { División de un } \\
\text { segmento por una } \\
\quad \text { razón dada }\end{array}$ & $\begin{array}{l}\text { Baricentro de } \\
\text { triángulo } \\
\text { Segmento divido } \\
\text { por una razón } \\
\text { dada } \\
\text { Operaciones con } \\
\text { vectores. }\end{array}$ & $\begin{array}{l}\text { Baricento del } \\
\text { triangulo } \\
\text { Segmento en forma } \\
\text { paramétrico } \\
\text { Operaciones con } \\
\text { vectores: igualdad, } \\
\text { adición y } \\
\text { paralelismos. } \\
\text { Combinación lineal. }\end{array}$ & $\begin{array}{l}\text { Baricentro del } \\
\text { triángulo } \\
\text { Rectas en forma } \\
\text { paramétrica } \\
\quad \text { Sistema de } \\
\text { ecuaciones }\end{array}$ & $\begin{array}{l}\text { Baricentro del } \\
\text { triángulo } \\
\text { Planos en forma } \\
\text { cartesiana } \\
\text { Sistema de } \\
\text { ecuaciones } \\
\text { Matrices y } \\
\text { Determinante } \\
\text { Regla de Cramer }\end{array}$ \\
\hline
\end{tabular}

Fuente: Los autores.

Por lo tanto, podemos identificar dos tecnologías para la demostración y son trabajar sin vectores o con vectores, como se muestra en la figura 12 .

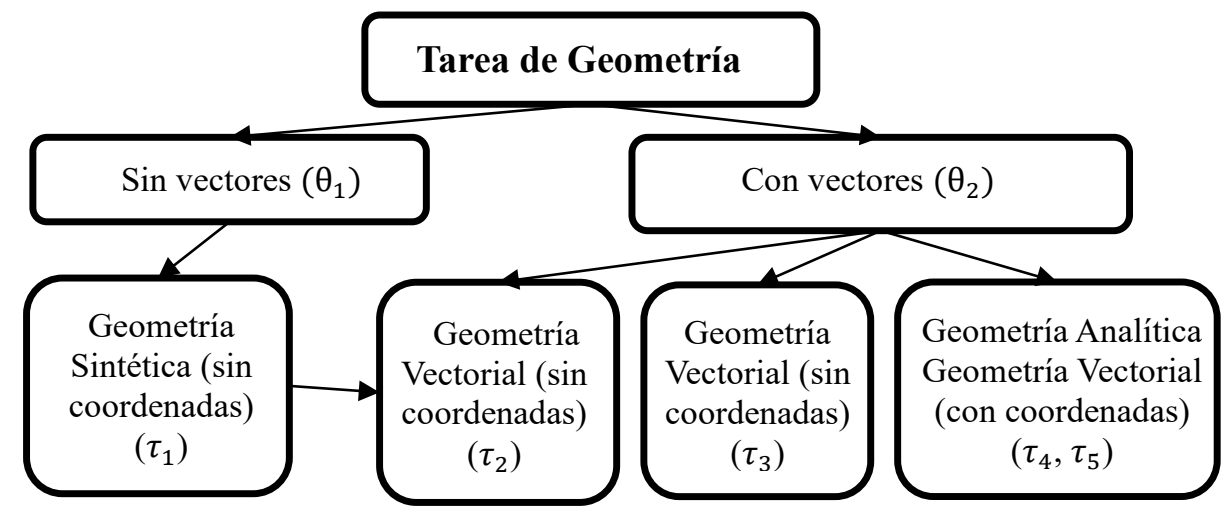

Figura 12. Tecnologías que justifican las técnicas para la resolución de la tarea. Fuente: Los autores.

\section{Conclusiones}

En este artículo se observó que la tarea de determinar el baricentro del tetraedro fue abordada por medio de cinco técnicas, donde se apreció que las demostraciones fueron hechas sin vectores (en geometría sintética) y con vectores (en geometría analítica y vectorial) y haciendo uso de las propiedades (tecnologías) respectivas en cada geometría (teoría).

De primera y segunda forma de haber sido realizado la demostración, podemos inferir que fue posible articular las geometrías para tener resultados explícitos. Además, la propiedad 
de división de un segmento por una razón dada permitió concluir la demostración. De la tercera, cuarta y quinta forma podemos inferir que debido a que presentan propiedades en común tales como combinación lineal, independencia lineal de vectores permiten concluir la demostración con vectores. Así mismo, se cuenta con una tecnología que reúne propiedades tanto geométricas como algebraicas que facilitaron las demostraciones.

Se puede notar que cada una de las técnicas $\left(\tau_{i}\right)$ presentadas se trataría de OMP y, al agrupar $\left(\tau_{1}\right)$ y $\left(\tau_{2}\right)$, es decir la que no usa coordenadas con la que sí se puede hablar de una OML. Asimismo, al articular las que usan coordenadas (con la tecnología $\left(\theta_{2}\right)$ ), da lugar a una OML. Finalmente, el estudio de la OM permitirá analizar y proponer la OD.

Para el objeto matemático baricentro tenemos que es enseñado tanto en la Educación Básica Regular (Programa curricular de Educación Primaria y Secundaria, 2018) como Educación Superior, en particular en el nivel universitario (Sumilla de Algebra Matricial y Geometría Analítica, 2018).

Por lo tanto, el MED que presenta el sistema educativo peruano se muestra en el siguiente esquema que podemos apreciar en la figura 13.

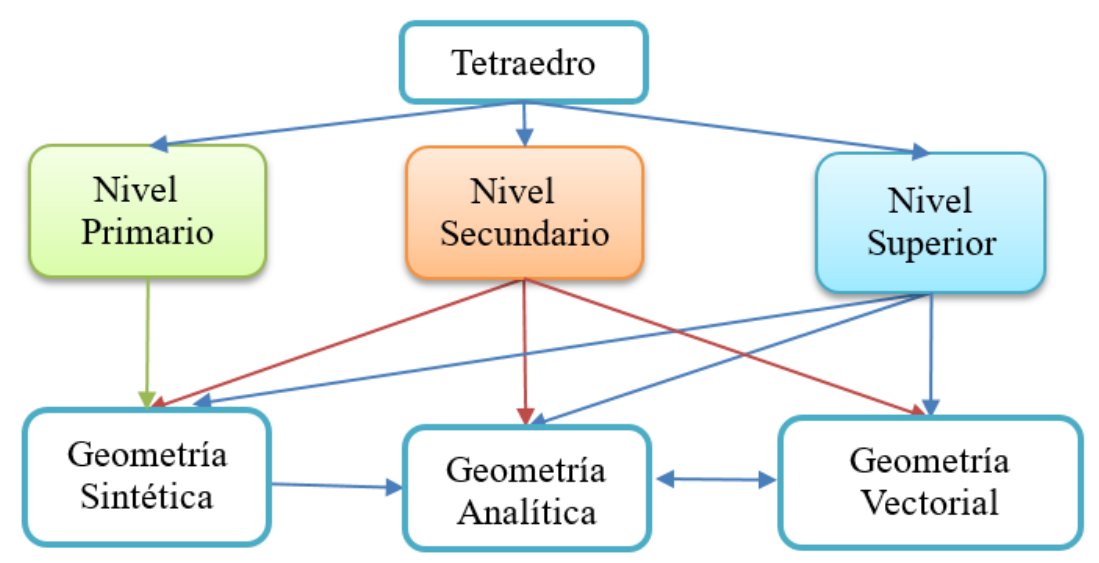

Figura 13. Modelo Epistemológico Dominante del objeto matemático Tetraedro Fuente. Los autores

En la figura 13 se puede apreciar que, en el contexto peruano, en el nivel primario se estudia la geometría sintética sin demostración del baricentro, de modo que puedan distinguir las diferentes figuras geométricas. En el nivel secundario se estudia la geometría sintética y vectorial con algunas demostraciones. Si bien se estudia propiedades como el baricentro del triángulo, rara vez las bimedianas y el baricentro del tetraedro en geometría analítica. En el nivel superior, es más frecuente realizar las demostraciones del baricentro del triángulo y muy pocas veces se estudia el baricentro del tetraedro en los cursos de geometría analítica y vectorial, ya que representa serias dificultades para los estudiantes. 


\section{Agradecimientos}

Agradecemos al Programa de Estudantes-Convenio de Pós-graduação - PEC-PG, da CAPES/CNPq - Brasil.

\section{Referências}

Almouloud, S., Regnier, J., \& Silva, C. (2009). Resolver problemas envolvendo Prova e demonstração: Uma dificuldade para professores de ensino básico. Proceedings of the $1^{\text {rst }}$ International Congress of Mathematics, Engineering and Society - ICMES 2009 Curitiba, Brazil.

Balacheff, N. (1987). Processus de preuve et situations de validation. Educational Studies in Mathematics, 18, 147-176.

Chevallard, Y. (1999). L'analyse des pratiques enseignantes en théorie anthropologique du didactique. Recherches en Didactique des Mathématiques, 19 (2), 221-265.

Gascón, J. (2003). La necesidad de utilizar modelos em didáctica de las Matemáticas”, Revista do Programa de Estudos Pós-Graduados em Educação Matemática, São Paulo, v. 5, n. 2, pp. 11-37.

Gascón, J. (2011). Las tres dimensiones fundamentales de un problema didáctico. El caso del álgebra elemental. Revista Latinoamericana de Investigación en Matemática Educativa, 14(2), 203-231.

Perú, Ministerio de Educación. Programa curricular de Educación Primaria, 2018 (1) Recuperado de: http://www.minedu.gob.pe/curriculo/pdf/programa-curricular-educacionprimaria.pdf.

Perú, Ministerio de Educación. Programa curricular de Educación Secundaria. 2018 (2) Recuperado: http://www.minedu.gob.pe/curriculo/pdf/programa-curricular-educacionsecundaria.pdf.

Sumilla de Algebra Matricial y Geometría Analítica (2018). Recuperado de : http://facultad.pucp.edu.pe/generales-ciencias/informacion-para-el-estudiante/sumillas/

Touré, S., Akele, C., Baye, B., Bendiman, K., Conde, K., Djiguiba, O., Don, A, Neulat, J. \& Traoré, T. (1997). Collectión Inter Africaine de Mathematique. 1erSM_Mathematiques. Paris, Francia. EDICEF.

Submetido em: 02/02/2019

Aceito em: 09/09/2019 